\title{
GESTIÓN EFICIENTE DEL CORREO ELECTRÓNICO: UNA EXPERIENCIA CORPORATIVA
}

\author{
Anna Sainz-Aloy y Cristina Soy-Aumatell
}
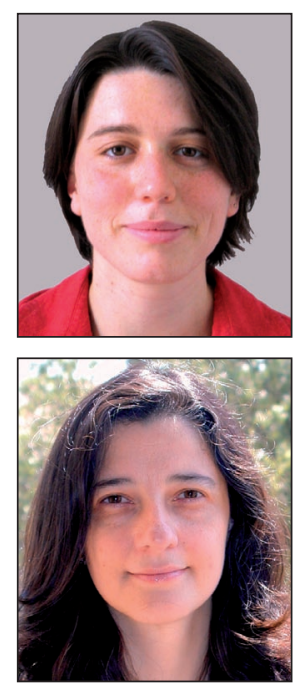

Anna Sainz-Aloy es diplomada en biblioteconomía y documentación por la Universitat de Barcelona y licenciada en documentación por la Universitat Oberta de Catalunya. Es documentalista del departamento Gestión de la Información de Criteria CaixaCorp.

Criteria CaixaCorp SA, Gestión de la Información Av. Diagonal, 621-629. 08028 Barcelona asainz@criteria.com

Cristina Soy-Aumatell, diplomada en biblioteconomía y documentación, es licenciada en geografía e historia y doctora en documentación por la Universitat de Barcelona (UB). Es responsable de Gestión de la Información de Criteria Caixacorp y profesora asociada de la UB.

Criteria CaixaCorp SA, Gestión de la Información Av. Diagonal, 621-629. 08028 Barcelona csoy@criteria.com

\section{Resumen}

La sobreinformación, y más concretamente la sobrecarga informativa y actividad derivada de la gran cantidad de correo electrónico que las organizaciones generan y reciben, repercute negativamente en su productividad, eficiencia, creatividad e innovación. Se presenta una experiencia práctica de mejora de la gestión del correo electrónico consistente en una serie de pautas destinadas a producir menos correo y de más calidad, y a administrar de forma más eficiente los buzones de correo individual y departamental. Se exponen los objetivos del proyecto, su implantación a través de talleres formativos y los principales resultados obtenidos.

\section{Palabras clave}

Sobreinformación, Correo electrónico, Estudio de caso, Gestión de la información, Productividad personal.

\section{Title: Efficient management of electronic mail: a corporate case study}

\begin{abstract}
Information overload, and more specifically the overload and associated activity due to the large amount of email received and issued in our organizations, affects business productivity, efficiency, creativity and innovation. This article presents a practical experience of improved email management based on the development of guidelines and tools addressed to creating less mail and more quality, and managing personal and departmental inboxes more efficiently. The project's goals, its implementation through workshops, and the main results achieved are reviewed.
\end{abstract}

\section{Keywords}

Information overload, Email management, Case study, Information management, Personal productivity.

Sainz-Aloy, Anna; Soy-Aumatell, Cristina. "Gestión eficiente del correo electrónico: una experiencia corporativa". El profesional de la información, 2011, septiembre-octubre, v. 20, n. 5, pp. 571-576.

http://dx.doi.org/10.3145/epi.2011.sep.12

\section{El problema de la sobreinformación o information overload}

El término sobreinformación se asocia a la situación de sobrecarga informativa en la que se dispone de más informa- ción para procesar de la que humanamente es factible asimilar, causando ansiedad. Aunque el término fue acuñado en 1970 por el sociólogo Alvin Toffler (1970) en su libro Future shock, se popularizó en España bajo el neologismo infoxicación por Alfons Cornella (1996). El tema de la infoxicación

Artículo recibido el 11-06-11

Aceptación definitiva: 25-06-11 
no es nuevo (Reuters, 1998) como ámbito de investigación dentro de los estudios de la conducta informativa, pese a que en los últimos años han aparecido estudios en publicaciones de referencia del ámbito empresarial internacional que amplían su alcance y elevan su perfil al plano tácticoestratégico de las empresas. Es el caso de las prestigiosas revistas McKinsey quarterly (Derek; Web, 2011), Harvard business review (Hemp, 2009) o el semanario económico The economist (2010), que han dedicado artículos al fenómeno de la sobreinformación. Estos trabajos analizan principalmente sus consecuencias tanto para personas como para empresas, posibles cambios organizativos a implementar, y cómo el buen uso de la tecnología puede ayudar a minimizar sus efectos.

La sobreinformación, así como la interrupción constante del trabajo de los profesionales a causa de la recepción de nueva información a través de múltiples canales y formatos (correos electrónicos, mensajería instantánea, mensajes de texto, foros, listas de distribución, alertas de todo tipo, RSS, llamadas telefónicas, prensa y revistas técnicas, petición de solicitud de informes y un largo etcétera) constituye un serio problema para las organizaciones. Disminución la productividad de los empleados, dificulta su creatividad e innovación e inhibe la aportación de valor; asimismo, afecta a la toma de decisiones de las organizaciones y al bienestar personal de sus empleados, dispersando la atención. Son numerosos los estudios publicados en los últimos años que abordan este problema en el contexto específico de las empresas y que ofrecen evidencias de su relevancia y de la necesidad de acometer acciones. Es el caso de las investigaciones conducidas por Basex, Microsoft o Intel, por citar algunos ejemplos.

La sobreinformación disminuye la productividad de los empleados y dificulta su creatividad e innovación

Basex (Spira, 2007), consultora americana especializada en los problemas descritos, ha cuantificado las pérdidas en productividad y reducción en innovación que la sobreinformación supone para la economía estadounidense. Sus investigaciones estiman que las interrupciones consumen un $28 \%$ de la jornada laboral, lo que equivale a unas 2 horas diarias, y revelan que un trabajador dedica sólo 11 minutos a un proyecto antes de que su concentración se vea interrumpida por algún correo, llamada o algún compañero.

Una investigación realizada por Microsoft indica que después de una interrupción por un correo electrónico, un empleado tarda 24 minutos en reconectar con una tarea. A menudo las interrupciones provocan la lectura de correo no abierto previamente o iniciar otras actividades no relacionadas, como por ejemplo navegar por internet.

Un estudio realizado entre más de 2.000 empleados de Intel señala que uno de cada tres correos recibidos por un empleado es innecesario y que la media de mensajes electrónicos ronda los 350 correos semanales por empleado, cifra que en el caso de los directivos puede ascender también a 300 diarios.

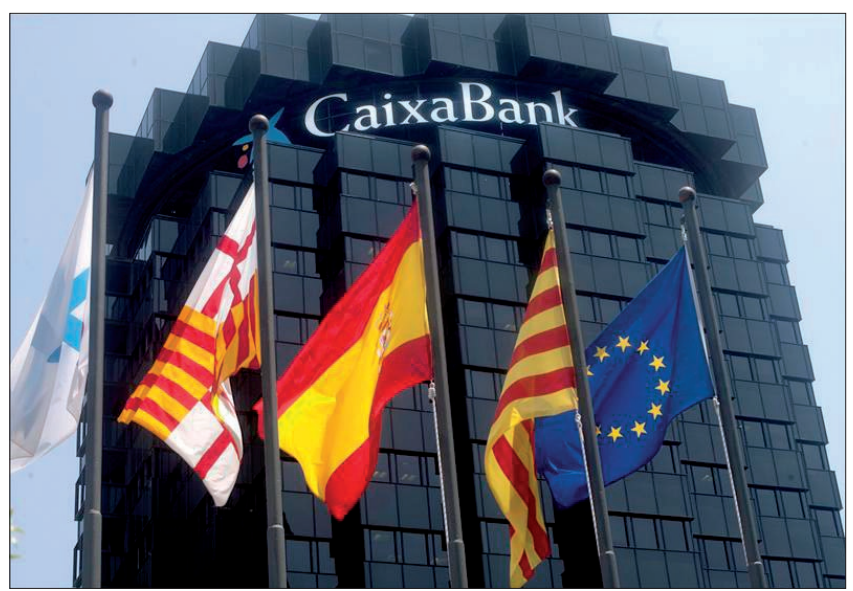

Edificio central de CaixaBank en la Avenida Diagonal de Barcelona

La consultora Linda Stone utiliza la expresión continuous partial attention para describir el estado mental de los trabajadores del conocimiento.

\section{La sobreinformación afecta a la toma de decisiones de las organizaciones y al bienestar personal de sus empleados}

\section{La experiencia en Criteria CaixaCorp}

Criteria CaixaCorp, que en julio de 2011 se convirtió en CaixaBank, el banco cotizado del grupo la Caixa, es un grupo inversor con participaciones en empresas financieras e industriales. La compañía tiene como accionista de referencia a la Caixa y cotiza en el mercado continuo de la bolsa española desde octubre de 2007. La trayectoria de Criteria/ CaixaBank se ha caracterizado por una apuesta firme por la internacionalización y la gestión activa de su cartera. A 31 de diciembre de 2010, Criteria contaba con la cartera de participaciones empresariales más importante de España en volumen total de activos brutos (GAV), con un valor de 23.059 millones de euros. El beneficio neto consolidado durante 2010 ascendió a 1.823 millones de euros, con un crecimiento del $38 \%$ respecto al ejercicio 2009 , y el beneficio recurrente neto consolidado se cifró en 1.424 millones de euros en el citado período, un $21 \%$ más que en el año 2009.

El departamento Gestión de la Información tiene entre sus líneas estratégicas de actividad la mejora de la eficiencia en el uso de la información y el incremento de la productividad de los empleados. El pasado 2010 puso en marcha un proyecto interno destinado a sacar partido de las herramientas y a aplicar pautas para optimizar la gestión de la información por parte de los empleados. Se trata de una iniciativa que incide en la gestión personal de la información, concepto que incluye cualquier input que llega al empleado en su rutina diaria independientemente de su formato o canal: papel (correspondencia, informes, revistas, prensa), medio electrónico (webs, archivos, correos, etc.) o de forma oral (llamadas telefónicas, consultas directas, reuniones...).

El proyecto surgió a iniciativa de un área de negocio de la compañía que solicitó al departamento de Gestión de la 
Información que le asesorase para mejorar la gestión del correo electrónico individual y departamental. A partir de esta petición se detectó la oportunidad de abordar una problemática que afecta a toda la compañía, y se trabajó en un conjunto de soluciones útiles y muy prácticas para los diferentes departamentos.

El correo electrónico en el entorno profesional concreto constituye una aplicación básica de comunicación interna, externa y corporativa; un útil de trabajo transaccional clave, a la vez que un importante repositorio y buscador documental que confirma que se trata de una herramienta imprescindible y muy crítica para la mayoría de empleados.

\section{Gestión de la Información tiene entre sus líneas estratégicas la mejora de la eficiencia en el uso de la información y el incremento de la productividad de los empleados}

Una de las primeras actividades en la puesta en marcha del proyecto consistió en medir la sobreinformación en la organización. Se diseñó un cuestionario y se seleccionó a un grupo heterogéneo de 19 empleados (un 20\% de la plantilla) a los que se entrevistó personalmente por espacio de unos 30-40 minutos para conocer sus hábitos en relación con el correo electrónico, la documentación recibida en papel y en soporte electrónico, su conocimiento de las herramientas de Microsoft Outlook (el programa de correo corporativo), el uso de dispositivos móviles - muy generalizados en la organizacióny, en general, su percepción de la sobrecarga informativa.

El análisis de las conclusiones obtenidas de las entrevistas reveló que el correo electrónico era la mayor preocupación de los empleados a la hora de gestionar su información personal. Ésta planteaba dificultades en mayor o menor grado en todos los departamentos, funciones, empleados con más o menos experiencia, responsabilidad y alfabetismo tecnológico e informacional. Los empleados tenían la percepción de recibir demasiados mensajes y, por lo general, desconocían las posibilidades de Outlook que podían resultarles de utilidad para gestionar el correo electrónico de forma más eficiente.

Las entrevistas también permitieron identificar algunas buenas prácticas llevadas a cabo por determinados departamentos, susceptibles de ser extendidas al resto de la organización.

\section{El correo electrónico como eje central del proyecto}

A partir de estas conclusiones, el Departamento de Gestión de la Información decidió centrar el ámbito del proyecto en la gestión del correo electrónico individual y departamental con el propósito de ofrecer al empleado herramientas o metodologías que le ayudaran a:

- disminuir el volumen de correos recibidos y enviados, además de mejorar su calidad;
- gestionar mejor el buzón de correo personal y las tareas que se derivan de su actividad;

- garantizar la conservación de los correos más críticos para la organización.

Estos tres objetivos se concretaron en el desarrollo y aplicación de una serie de pautas y métodos y, concretamente, en una email etiquette, unas recomendaciones para gestionar los buzones individuales y una serie de propuestas para la gestión del correo departamental que se detallan en los apartados sucesivos. También se aborda la gestión de buzones, acciones y tareas relacionadas desde dispositivos móviles Blackberry.

A partir del estudio de la bibliografía internacional sobre gestión personal de la información, mejora de la eficiencia y de la productividad personal y organizativa y, por extensión, de la atención, se identificaron algunos métodos reconocidos y aplicados por grandes empresas, que se adaptaron al entorno de la empresa, como GTD (Getting things done) desarrollada por el consultor David Allen, Inbox zero de Merlin Mann o la plasmada en The hamster revolution (Song, 2008).

Los empleados tenían la percepción de recibir demasiados mensajes y desconocían las posibilidades de Outlook útiles para gestionar el correo electrónico de forma eficiente

\section{Email etiquette}

Con el objetivo de generar menor volumen de correo y de mayor calidad, se elaboró una lista de normas básicas, basadas en el sentido común, a considerar cuando se redacta y se envía correo electrónico.

Las reglas de email etiquette propuestas, a modo de resumen son:

- Responder siempre. Cuando no sea posible responder un correo en un tiempo razonable, confirmar al remitente cuándo se tiene previsto responder. Ello evitará correos de reclamación innecesarios.

- Especial atención al Asunto. Escribir un texto claro, conciso e informativo, siempre igual para un mismo tema. Ello facilitará la búsqueda e identificación de los correos. Lo mismo se aplica al texto. Corregir errores tipográficos $u$ ortográficos. No mezclar distintos temas o asuntos en un mismo correo.

- Evitar el ping-pong. Minimizar el correo de múltiples envíos y respuestas. Si la constancia escrita no es importante, llamar por teléfono para reducir el número de correos intercambiados, o utilizar incluso la mensajería instantánea (alternativa para algunas comunicaciones si la situación lo requiere o prevemos que puede desencadenar una cadena de emails que puede solventarse descolgando el teléfono).

- Limitar el uso del Responder a todos, utilizando esta opción con precaución. Ser selectivo al elegir los destinatarios a poner en copia ( $\mathrm{CC} / \mathrm{CCO})$. Así se contribuye a redu- 
cir el número de correos que reciban otras personas, que a su vez pueden ser emisores de nuevos mensajes.

- Revisar lo que se reenvía. Al reenviar un correo se debe añadir algún comentario para que quien lo reciba sepa si debe actuar en algún sentido o si se remite sólo a título informativo (FY). Puede ser útil cambiar el texto del Asunto y editar el mensaje original eliminando contenido innecesario.

- Enviar menos correos. Es una de las recomendaciones más importantes. La regla de oro es: cuantos menos envíes, menos recibirás.

Los talleres se han hecho extensivos a todos los empleados de la compañía y actualmente se integran en el plan de formación interno

\section{Gestión eficiente del buzón de correo electrónico}

Para gestionar el buzón de correo individual se elaboraron unas directrices que sirven de guía en la toma de decisiones acerca del correo entrante y saliente, y la forma óptima de organizar los buzones.

El método consiste en dejar a cero la bandeja de entrada del buzón de correo electrónico, y gestionar los correos sobre los que realizar acciones pendientes mediante carpetas de acción.

Estas carpetas son claves para el buen funcionamiento del método. Se proponen inicialmente tres carpetas imprescindibles: @POR HACER; @MEDEBEN; @POR LEER. Estas carpetas deben ser perfectamente identificables en la estructura personal de carpetas, por eso se encabezan por una @ y se utilizan mayúsculas. Las carpetas de acción servirán para ir moviendo los correos que entran o salen del buzón según requieran una acción (@POR HACER), alguien deba decir algo al respecto (@ME DEBEN) o haya que revisarlos más tarde (@POR LEER), teniendo en cuenta que todos los correos que resulten de fácil solución, que lleven menos de 2 minutos contestar, se deben liquidar lo antes posible al recibirlos. Cada persona deberá encontrar las carpetas que más le encajen, quizás necesitará otras del tipo: Urgente, Jefe..., etc.

Lo más importante es ser expeditivo al recibir los mensajes. Al instante de recibirlos deben:

- eliminarse si no sirven;

- moverse a una carpeta de acción;

- responderse, si requieren menos de 2 minutos;

- clasificarse en carpetas de archivo si ya se han procesado y se considera que deben conservarse.

Lógicamente este método requiere revisar regularmente las carpetas de acción (activar alertas sobre estos mensajes si se considera necesario) e intentar automatizar al máximo los procesos recurrentes utilizando las reglas y otras herramientas disponibles en Outlook.

\section{Cómo hacer que el correo electrónico trabaje porti}

Al margen de la aplicación de la email etiquette y de las directrices para gestionar de forma eficiente el buzón de correo individual, Outlook tiene numerosas funciones para que el correo trabaje por nosotros de forma automática. A continuación se identifican aquellos trucos u opciones que pueden ayudarnos a contener el tamaño de los buzones:

- Gestor de tareas: permite planificar tareas, delegarlas, activar alertas, etc.

- Asignación de categorías: facilita la clasificación de elementos de Outlook de forma temática y distinguirlos visualmente mediante colores además de filtrarlos en búsquedas.

- Creación de reglas automáticas: permite establecer de manera automática las condiciones de archivado, eliminación o reenvío de los correos entrantes y salientes.

- Activación del botón "No guardar": muy útil para mantener limpia la carpeta de correo enviado, al poder decidir si se guarda o no un correo al momento de crearlo.

- Uso del botón "Guardar elemento enviado": permite decidir en qué carpeta guardaremos un correo antes de enviarlo.

- Búsquedas en Outlook, para conocer a fondo todas las funcionalidades de búsqueda dentro de nuestro buzón.

- Control del correo electrónico no deseado.

Estas herramientas ayudan a reducir el tiempo dedicado a la revisión de los buzones, a eliminar y a mover correos.

Entre los beneficios tangibles destaca que se genera menos correo y que las acciones vinculadas a éste están más organizadas

\section{Gestión del correo a nivel departamental}

Una vez abordado el problema de la gestión individual del correo, se plantearon posibles soluciones para gestionarlo de forma conjunta a nivel departamental o de grupos de trabajo integrados por varios profesionales.

El departamento que solicitó el proyecto estaba interesado en mejorar la gestión de correos comunes a todo el equipo, duplicados en distintos buzones de Outlook, y en garantizar la conservación y el acceso a todos los correos críticos, sobre todo aquellos vinculados a proyectos de inversión y a la actividad de control de gestión.

Las alternativas disponibles teniendo en cuenta la tecnología implantada en Criteria eran tres, cada una con sus ventajas e inconvenientes:

- Transferir los correos más relevantes al gestor documental corporativo Microsoft Sharepoint 2007. La mayor ventaja era la de integrar como un documento más el correo dentro del gestor documental, unido a las potentes herramientas de descripción y búsqueda de Sharepoint. Como principal desventaja, se constató el bajo uso del gestor por parte de los miembros de la organización. 


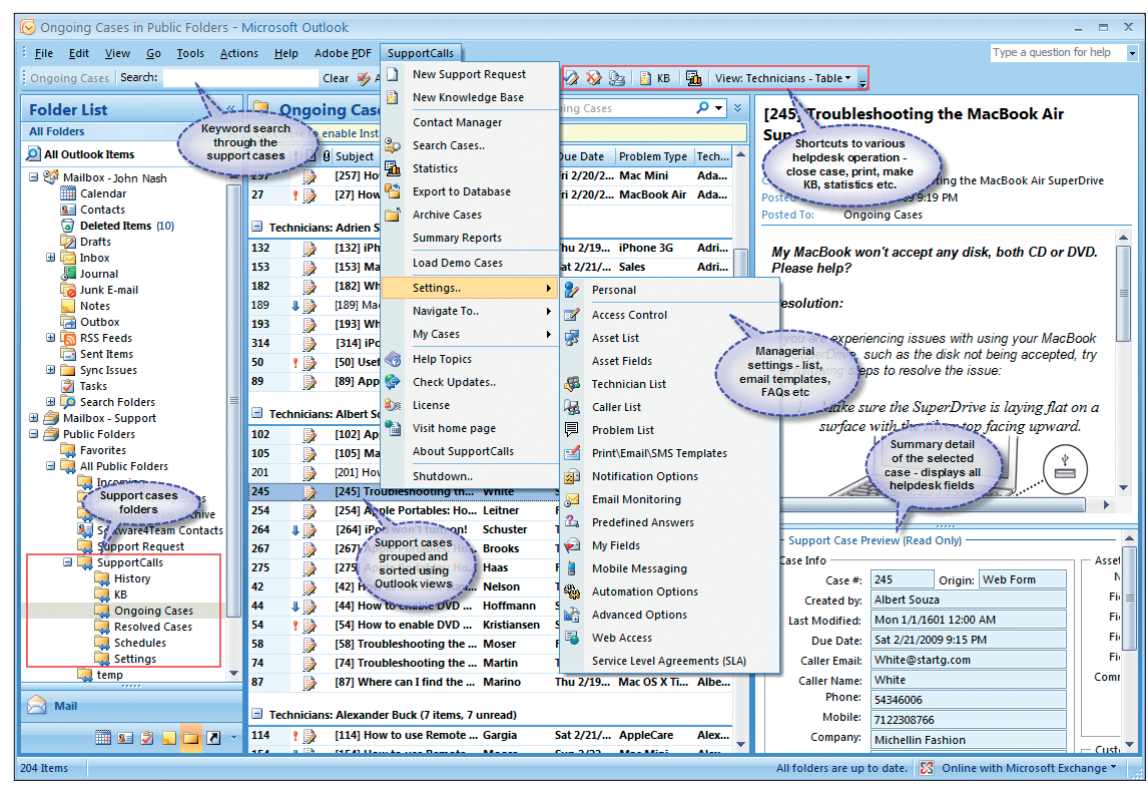

MS Outlook puede adaptarse a multitud de entornos de trabajo. En la imagen se muestra aplicado a un servicio de soporte técnico a clientes. http://www.fileguru.com

- Crear un buzón en Outlook compartido por todos los miembros del equipo. La ventaja radicaba en la facilidad a la hora de mover documentos entre buzones, así como en las potentes funciones de búsqueda de Outlook de forma integrada con los correos de los buzones personales de cada miembro del equipo.

- Guardar los correos en las carpetas comunes del servidor corporativo. La ventaja estaba en poder guardar los correos de forma integrada con el resto de documentos de cada proyecto (informes, contratos, etc.); como desventaja importante se destacó la pérdida de funciones de búsqueda de los correos mediante el simple buscador de escritorio.

Después de valorar cada una de las opciones, se consideró que la mejor era la segunda: utilización de buzones compartidos en Outlook, una elección de muy fácil implantación.

\section{Prueba piloto}

Para testar la utilidad y oportunidad de los métodos elaborados, el Departamento de Gestión de la Información realizó una prueba piloto durante tres semanas que posteriormente se hizo extensiva a toda el Área de Organización y Sistemas de Información, formada por usuarios muy avanzados en ofimática. En total participaron en esta prueba 7 personas.

Las conclusiones obtenidas de esta fase fueron:

- Menor archivo de correo. Se reduce significativamente el tamaño de los buzones personales de Outlook.

- Mayor control sobre las acciones pendientes derivadas de cada correo que se recibe o se envía.

- Reducción del tiempo dedicado a gestionar el correo debido a una mayor automatización de procesos (activación de reglas, asignación de categorías, etc.).

- Motivación por encontrar soluciones a problemas de Outlook que hasta el momento se consideraban irresolubles.

- El hecho de disponer de una bandeja de entrada vacía aporta sensación de tranquilidad e "higiene mental" al empleado, reduciendo el estrés derivado de ver grandes cantidades de mensajes por procesar.

\section{Talleres formativos}

Una vez probada la metodología y contrastada con una serie de usuarios clave se valoró cual resultaría la mejor fórmula para implantarlo en toda la organización. Se propuso hacerlo en forma de talleres formativos. Los talleres se plantean por áreas organizativas, bajo un formato muy práctico, y son altamente participativos. Fueron un punto de encuentro en el que los asistentes pudieron compartir necesidades y dificultades comunes, mostrando experiencias y soluciones a los problemas de cada empleado con el correo electrónico. Cada sesión fue precedida de otra reunión preparatoria con el responsable del departamento o en quien éste delegara, en la que se planteaban casos concretos para tratar en el taller. Una vez impartidas las sesiones se realizan acciones de soporte y de seguimiento. Los formadores resuelven dudas y proporcionan asistencia y asesoramiento para la implantación de las pautas y métodos propuestos.

La primera de las áreas en participar en un taller formativo fue precisamente la que solicitó el proyecto (cliente interno del proyecto) formada por un equipo de 10 personas, todas ellas dedicadas al control de gestión. En el taller se trataron las metodologías destinadas a mejorar la gestión de los buzones individuales, explicando el funcionamiento de algunas herramientas útiles de Outlook y planteando posibles escenarios para gestionar el correo departamental, creando un buzón compartido en Outlook. Los asistentes llevaron sus portátiles y salieron de la sesión con parte del trabajo hecho y motivados para aplicar las posibles mejoras.

Los talleres, impartidos por los profesionales de gestión de la información de la compañía se han hecho extensivos a todos los empleados de la compañía y actualmente se integran en el plan de formación interno. Hasta la fecha, aproximadamente 70 empleados ( $70 \%$ de la plantilla) ya ha participado en estas sesiones.

\section{Gestionar bien el correo electrónico con-} tribuye a que las personas sean más productivas y los procesos más eficientes

\section{Conclusiones}

La iniciativa ha tenido una buena acogida entre los empleados, debido a que el correo electrónico es una herramienta de la que todos somos usuarios habituales e intensivos. Si bien no se ha realizado una evaluación formal del grado de adopción, pues está aún implantándose, el interés por participar en los talleres y las cuestiones y dudas posteriores a las sesiones nos permiten afirmar que las pautas y métodos se están aplicando. 
Las pautas y recomendaciones proporcionadas no constituyen políticas corporativas, sino que son un conjunto de directrices lo suficientemente amplias y flexibles para que cada persona pueda adaptarlas a su forma de trabajo particular. Están alineadas con otras iniciativas relacionadas con la gestión de la documentación integral tanto de las diferentes áreas de negocio como transversales. Los talleres han contribuido a sensibilizar a los empleados sobre el tema y a compartir experiencias y mejoras.

Según datos de la Association for Information and Image Management, AIIM (2009), sólo un $10 \%$ de las organizaciones tienen política de correo electrónico, si bien un 20\% está trabajando en ello, y un $29 \%$ tenía previsto implantar una en los próximos dos años.

Entre los beneficios tangibles de la experiencia destaca que se genera menos correo y que las acciones vinculadas al correo están más organizadas. Los buzones departamentales, acompañados de una buena organización de los equipos de trabajo, son una solución para compartir correos entre los integrantes y permite localizar rápidamente los que son críticos para la actividad de la compañía. Outlook es una buena herramienta para localizar mensajes, contactos, tareas, citas, etc., de varios buzones proporcionando una visión integral de las relaciones intra grupo, con otras organizaciones, personas, por temas, fechas etc.

Gestionar bien el correo electrónico, dado el carácter nuclear de éste en nuestras organizaciones, contribuye a que las personas sean más productivas, utilicen la información de forma más efectiva y los procesos sean más eficientes.

\section{Bibliografía}

Allen, David. Getting things done. http://www.davidco.com/about-gtd

Cornella, Alfons. "Cómo darse de baja y evitar la infoxicación en internet". Extra!-net, 16-12-1996, n. 187.

http://www.infonomia.com/pdf/1996_12_16_extranet.187. infoxicacion.pdf

Cornella, Alfons. Infoxicación: buscando un orden en la información. Barcelona: Infonomia, 2009.

Derek, Dean; Webb, Caroline. "Recovering from informa- tion overload". Mckinsey quarterly, 2011, January, pp. 1-9. http://www.mckinseyquarterly.com/Recovering_from_in formation_overload_2735

E-mail management. AlIM, 2009.

http://www.aiim.org/email

Hemp, Paul. "Death by information overload". Harvard business review, 2009, September, pp. 83-89.

Information overload research group. http://iorgforum.org/

Intelligence, intuition and information: a Thomson Reuters white paper. New York: Thomson Reuters, 2010.

http://thomsonreuters.com/content/corporate/docs/Intel ligence_Intuition_and_Information.pdf

Kevin, Miller. Surviving information overload: the clear, practical guide to help you stay on top of what you need to know. Grand Rapids, Mich.: Zondervan, 2004. ISBN: 031025115X.

Mann, Merlin. Inbox zero.

http://inboxzero.com/articles

Out of the abyss: surviving the information age. London: Reuters, 1998. ISBN: 9780901249074.

Pollar, Odette. Surviving information overload: how to find, filter, and focus on what's important. Crisplearning, 2004. ISBN: 1560526947.

Song, Mike; Halsey, Vicki; Burress, Tim. La invasión de los emails: domina tu correo electrónico antes de que él te domine a ti. Barcelona: Integral, 2008. ISBN: 9788498672091. http://www.hamsterrevolution.com

Spira, Jonathan. Information overload: we have met the enemy and he is us. New York: Basex, 2007.

http://www.basex.com/web/tbghome.nsf/pages/ios

Stone, Linda. Continuous partial attention. http://lindastone.net/qa/continuous-partial-attention

The economist. "Data, data everywhere: a special report on managing information", 2010, 25 $5^{\text {th }}$ February, pp. 3-16. http://www.economist.com/node/15557443

Tofler, Alvin. Future shock. New York: Random House, 1970. ISBN 9780553277371.

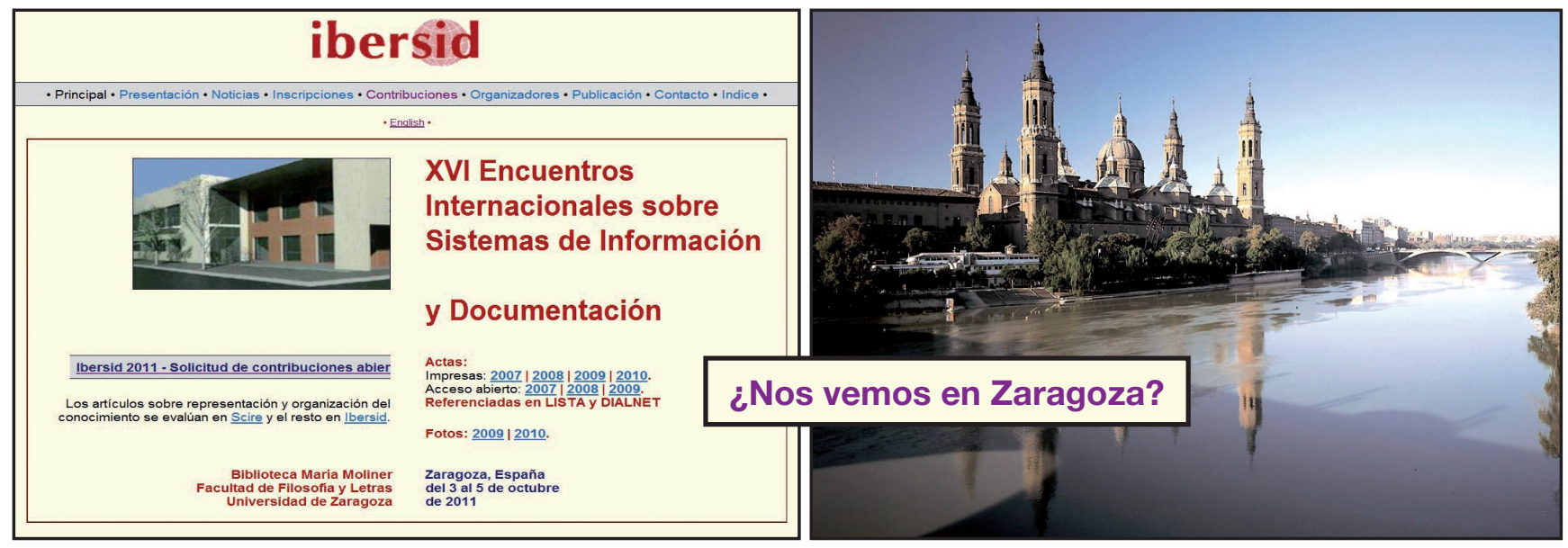

